\title{
Theoretical BER Evaluation of Passive RFID Tag-To-Tag Communications
}

\author{
Tarik Lassouaoui ${ }^{1}$, Florin Hutu ${ }^{1}$, Yvan Duroc ${ }^{2}$ and Guillaume Villemaud ${ }^{1}$ \\ ${ }^{1}$ Univ. Lyon, INSA Lyon, Inria, CITI, F-69621 Villeurbanne, France \\ ${ }^{2}$ Univ. Lyon, UCBL, Ampere Laboratory, F-69622 Villeurbanne, France
}

\begin{abstract}
The concept of UHF RFID passive tag-totag communication has been introduced and opens new promising perspectives in the field of Internet-of-Things. This concept enables two powerless tags to exchange information using an external source. Moreover, a simulation framework has been proposed as a tool allowing the performance evaluation of tag-to-tag radio links in terms of Bit Error Rate (BER). The aim of this paper is to provide a theoretical framework for BER estimation. The theoretical framework takes into account various parameters such as modulation rate, receivers topology and the electromagnetic coupling of the tags. Furthermore, in order to validate the obtained results, they are compared with the ones obtained by realistic simulations.
\end{abstract}

Index Terms-RFID tags, UHF antennas, Bit error rate, Amplitude shift keying, Demodulation.

\section{INTRODUCTION}

Radio frequency identification (RFID) is still a fast emerging technology with numberless applications especially in the areas of security, logistics or traceability [1]. RFID is also an evolving technology since new capabilities such as sensing, energy harvesting, spatial identification are added [2][3]. Another example of this tendency is the development of inter-tag communication concept for applications where data rates can be low and the receiver tag uses passive RF part (i.e. envelope detector)[4].

The concept of tag-to-tag (T2T) communication between two passive UHF RFID tags has already been proposed and experimentally validated in [5] for short distances. The main idea is to establish communication between two passive tags placed in close proximity. Energy feeding of these tags is achieved by an external source which can be a classical RFID reader or any other source providing a power level large enough in order to activate the tags. The communication between the tags is performed by backscattering modulation of the incoming RF sources such as WiFi signals, TV transmissions [4] and Bluetooth 4.0 Low Energy standard [6].

However, since the first studies few works have been done about this topic. In [7], the design of a power efficient demodulator is investigated to enable the tag-to-tag link to operate at long distances with a modulation depth as low as $75 \%$. In [8], a novel multiphase backscattering technique is proposed to overcome phase cancellation and demonstrating the capability of tag-to-tag link to operate at long distances (above $3 \mathrm{~m}$ ) under high excitation signal.

All things considered, these efforts mostly focus to deal with the performance limitations of the passive UHF RFID systems, like tag characteristics in terms of antenna gain polarisation and impedance matching, sensitivity of the receiver which defines the minimum level of the tag signal and propagation environment [9].

This paper focuses on the evaluation of the passive T2T communication in terms of bit error rate (BER) as traditionally done for evaluating wireless transmission systems. The performance depend on several parameters: i) electromagnetic coupling (i.e., the mutual coupling between the tags or the orientation of the tags with respect to the source); ii) modulation depth; iii) receivers topology (coherent or non-coherent demodulation). The general objective is to achieve a global optimization of the communication link between the tags. More precisely, antenna or radio front-end considerations may be extracted by such kind of analysis.

The proposed approach is based on the simulation framework discussed in [10]. The main idea is to consider the antennas of the two tags (LT -listener tag and RT reader tag) as an array of two mutually coupled radiating elements. With respect of the orientation of the illuminating source relative to the tags plane, the far field gain of the antenna array is varying. Moreover, the impedance provided by the LT antenna varies with respect of the distance between the two tags. The gain and impedance modification has an impact on the modulation depth and consequently on the quality of the communication. As stated, the present work gives for ASK modulation the analytical formula for the probability of error taking into account the particular case.

The rest of the paper is organized as follows. Section II describes more in detail the concept of tag-to-tag (T2T) communication with versatile modeling tools are required to understand and master the design of interacting-oriented tags. The theoretical investigation aimed to rewrite bit error probability according to crucial parameters that improve the range is detailed in section III. Finally conclusion and perspectives are given in section IV. 


\section{TAG-TO-TAG SIMULATION FRAMEWORK}

A T2T communication requires an external electromagnetic source in the employed frequency band that delivers a signal with a power high enough to ensure the tags activation. The information exchange is performed based on the same source by using the retro-modulation principle.

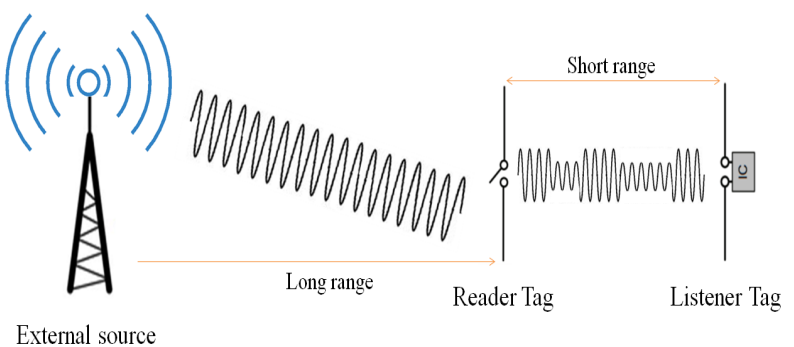

Fig. 1. General principle of tag-to-tag communication.

As mentioned previously, the RT will modulate the incoming signal to send a message towards the LT (Fig. 1). From the electromagnetic field perspective, the two tags may be described as an array of two mutually coupled antennas. The resulting antenna gains and the impedance variation of the LT antenna with respect of the RT variation are extracted from an electromagnetic field simulation performed with CST microwave studio software.

Then, system-level simulations are performed using Keysights Advanced Design System (ADS) software. The synoptic of the simulation framework is depicted in Fig. 2. As can be remarked, the external source (here a $\mathrm{CW}$ ) is modulated in amplitude (ASK) by switching between the two gains and impedance of the antennas $\left(G_{o n}, Z_{\text {on }}\right.$ and $G_{o f f}, Z_{\text {off }}$ respectively). The switch is performed in the rhythm of the data sent from the RT towards the LT.

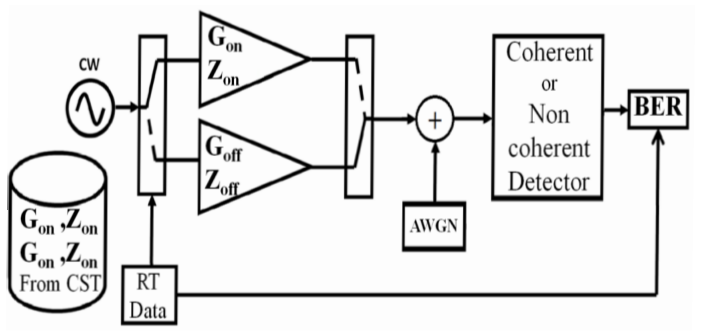

Fig. 2. Synoptic of the tag-to-tag system-level simulations.

\section{ANALYTICAL FORMULATION OF BER}

The variation in terms of antenna array gain and the impedance presented at the input of the LT, can be associated to a variation of the amplitude of the $\mathrm{CW}$ signal, at the rhythm of the data sent by the RT. In order to receive the data, on the LT, a demodulation stage is employed. The demodulation may be coherent or non-coherent. For the two cases, analytical expression of the probability of error with respect of signal to noise ratio can be developed [11][12].

The probability of error Pe in the case of a coherent and non-coherent reception is given below.

\section{A. Coherent detection}

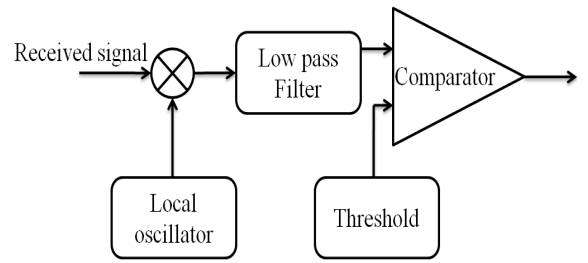

Fig. 3. Coherent ASK receiver.

The coherent or synchronous demodulation requires a carrier recovery circuit to transpose the received signal to the low frequencies. A block diagram of coherent detection is shown in Fig. 3. In this circuit, a local oscillator is tuned to oscillate at the same frequency as the received signal, the received RF signal multiplies with the local oscillator through an RF mixer generating the sum and the difference frequencies between them. The lowpass filter is used to recover the bandpass signal by eliminating the sum frequency component .

$$
P_{e, A S K C}=\frac{1}{2} \cdot \operatorname{erfc}\left(\sqrt{\frac{d^{2}}{4 N_{0}}}\right)
$$

The $\operatorname{erf} c$ function is given as:

$$
\operatorname{erfc}(x)=\frac{2}{\sqrt{\pi}} \int_{0}^{x} e^{-t^{2}} d t
$$

\section{B. Non coherent detection}

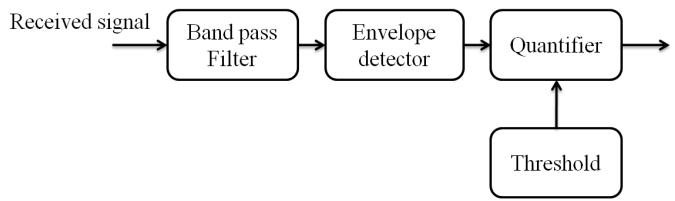

Fig. 4. Non-coherent ASK reciever.

The asynchronous ASK detector consists of a bandpass filter to reduce noise outside the frequency band, an envelope detector as a half-wave rectifier used to recover the baseband signal and a quantifier which delivers a digital output. The non-coherent detection does not require a synchronization mechanism, in the presence of noise that disturbs the signal, a system using non-coherent detection has a lower BER than coherent detection.

$$
P_{e, A S K N C}=\frac{1}{2} \cdot \exp \left(-\frac{d^{2}}{4 N_{0}}\right)
$$


Where $d$ corresponds to the difference between the amplitudes of the high and low bit states, and $N_{0}$ is the noise power spectral density.

$$
E_{b}=\frac{1}{2} \cdot\left(\frac{A_{1}^{2}}{2} \cdot T_{s}+\frac{A_{0}^{2}}{2} \cdot T_{s}\right)
$$

The average energy per bit $E_{b}$ is defined by the amplitudes of the two states $A_{0}, A_{1}$ of the binary flow and bit time $T_{s}$.

$$
A_{1 / 0}=A \cdot G_{\text {on } / \text { off }}\left(1-\left|\frac{Z_{\text {on } / \text { off }}-Z_{0}}{Z_{\text {on } / \text { off }}+Z_{0}}\right|\right)
$$

It is worth noticing that the modulation depth $M$ depends on the gain and switching impedance together with the received power coming from the external source.

$$
M=\frac{A_{1}-A_{0}}{A_{1}}
$$

For binary modulation schemes, we have two signals $S_{1}(t)$ and $S_{0}(t)$.

$$
\begin{gathered}
S_{1}(t)=\sqrt{\frac{2 E_{b}}{\beta}} \cdot \varphi(t) \\
S_{0}(t)=\alpha \cdot S_{1}(t)
\end{gathered}
$$

With :

$$
\begin{gathered}
\beta=G_{\text {on }}\left(1-\left|\frac{Z_{\text {on }}-Z_{0}}{Z_{\text {on }}+Z_{0}}\right|\right)+G_{\text {off }}\left(1-\left|\frac{Z_{\text {off }}-Z_{0}}{Z_{\text {off }}+Z_{0}}\right|\right) \\
\varphi(t)=\sqrt{\frac{2}{T_{s}}} \cdot \cos \left(2 \pi f_{c} t\right) \\
d=\sqrt{\frac{2 E_{b}}{\beta}} \cdot(1-\alpha)
\end{gathered}
$$

The parameter $d$ is strictly related to the average energy per bit $E_{b}$ which is defined according to the amplitudes of the two states $A_{0}, A_{1}$ of the binary flow and modulation rate $\alpha=\frac{A_{0}}{A_{1}}$.

By replacing the term $d$ in (1) and (3) with (11), we obtain:

$$
P_{e, A S K C}=\frac{1}{2} \cdot \operatorname{erfc}\left(\sqrt{\frac{(1-\alpha)^{2}}{\beta} \cdot \frac{E_{b}}{2 N_{0}}}\right)
$$

The $\operatorname{erf} c$ function is defined in (2).

$$
P_{e, A S K N C}=\frac{1}{2} \cdot \exp \left(-\frac{(1-\alpha)^{2}}{\beta} \cdot \frac{E_{b}}{2 N_{0}}\right)
$$

$\frac{E_{b}}{N_{0}}$ is the signal to noise ratio (SNR) for a digital signal, its a normalized version of SNR for a defined bandwidth and bit rate, used as a figure of merit.

\section{Simulation Results}

By using the simulation framework previously described, BER variation has been calculated. The simulations were performed considering the LT and RT illuminated by a CW at $868 \mathrm{MHz}$ and with a power level of $-50 \mathrm{dBm}$.

In order to have good accuracy for the obtained BER values, the number of transmitted bits was $10^{6}$. The bit time was set to 50 nsec. The gain and the coupling impedance value are defined to have several modulation depths ranging from $10 \%$ to $100 \%$. Without loss of generality, the simulation considered the impedance matching case (where $Z_{\text {on }}=Z_{\text {off }}=50 \Omega$ ) together with a missmatch case.

The BER value is estimated by comparing the base band signal of RT with the recovered signal of LT using synchronous or asynchronous demodulation, the digital version is recovered with a decision circuit after the demodulation.

As can be seen from Fig. 5 and Fig. 6, for a coherent and non-coherent detection, by assuming that the signal collected by the listener tag is degraded by AWGN. The performance of the T2T is proportional to the modulation depth that must be maximized with a proper impedance matching strategy accounting for the electromagnetic coupling among tags.

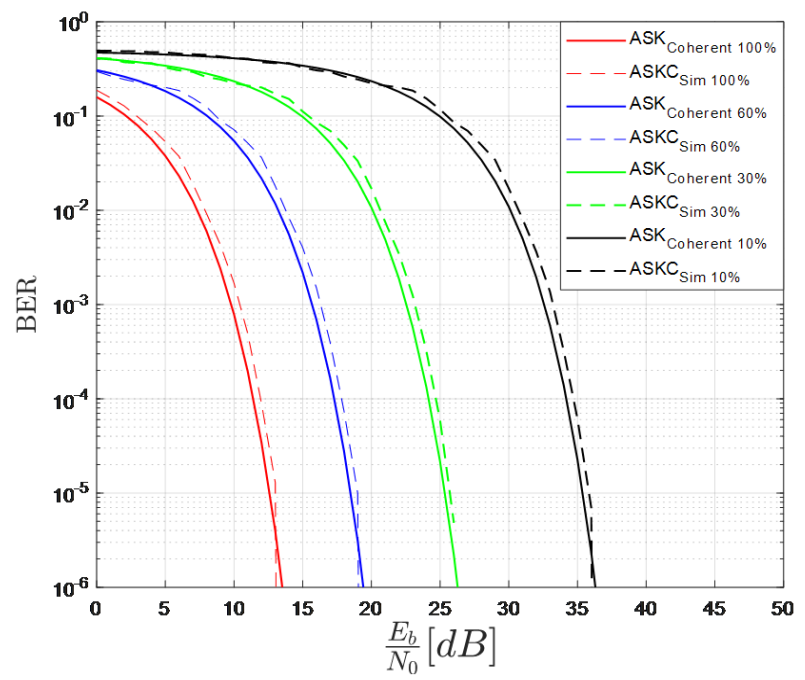

Fig. 5. Coherent demodulation: BER variation for different modulation depths in matched case.

We focus on the non coherent detection results that is used in passive T2T system for simplified circuitry and very low power cost.

In Fig. 6, for a bit error rate of $10^{-5}, A S K_{N C 100 \%}$ needs a signal to noise ratio of approximately $13 \mathrm{~dB}$ while $A S K_{N C 50 \%}$ is around $19 \mathrm{~dB}$. The reason is $A S K_{N C 50 \%}$ expends energy in both symbols used for transmission, but the symbols are alike and distance between them in signal 
space is small called modulation depth, the receiver is not able to make the difference between two binary levels in low signal to noise ratio.

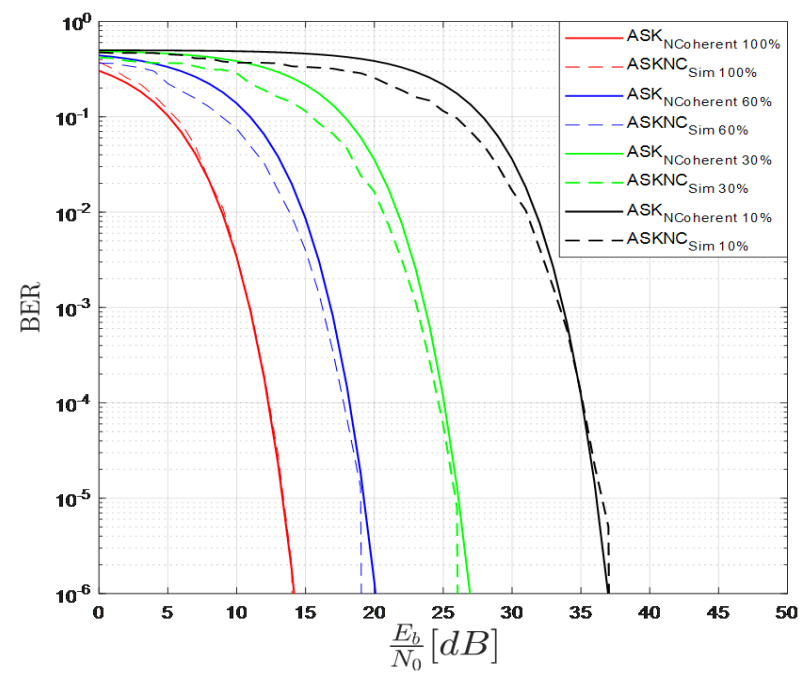

Fig. 6. BER variation for non-coherent demodulation for different modulation depths in matched case.

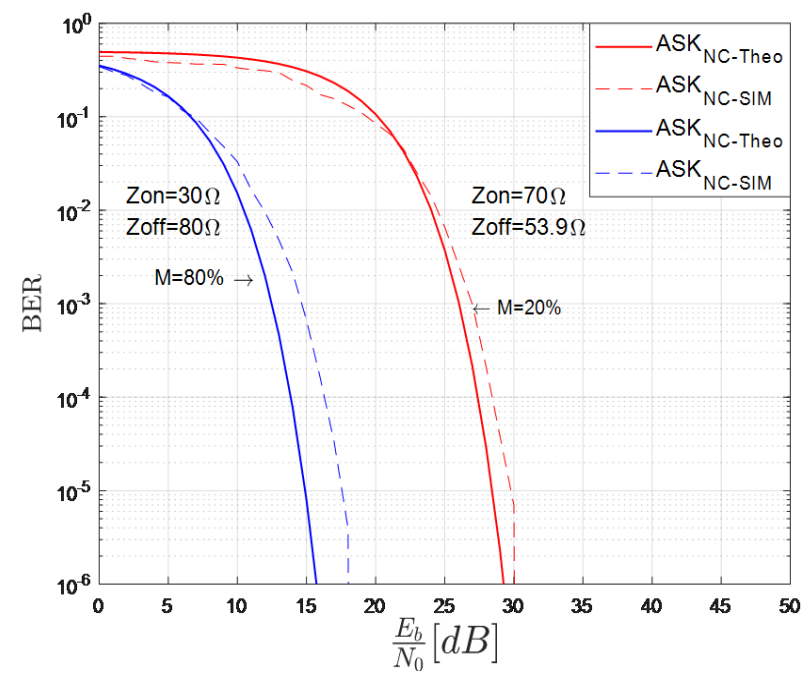

Fig. 7. BER variation for non-coherent demodulation in the miss-match case.

The difference between the theoretical results and the simulation in Fig. 7 comes from the way in which the BER and SNR is determined in simulation (statistical computation), while in MATLAB we define an interval for the SNR in which the theoretical BER is ploted.

\section{CONCLUSION}

This paper presents a theoretical background for the analysis of a passive tag-to-tag communication. BER evaluation demonstrates the importance of modulation depth, which is defined by the gain, coupling impedance and received power from the external source. Concluding that, the distance between the tags, their orientation and the position of the source play a crucial role in performance limitations of passive tag-to-tag systems and that the theoretical formulation will help to design an efficient T2T system.

A further study will be performed by completing the theoretical study, the aim is to make simulation framework as realistic as possible by considering several parameters like electromagnetic coupling (i.g. complex coupling impedance) and different tags configuration where the antennas are spaced, staggered and arbitrarily oriented. Also, by adding scenarios where the external source is a complex RF waveform from, using a more realistic radio channel and receivers with passive circuits. This will leads us to measure the maximum RT-to-LT tag communication distance in a variety of controlled scenarios.

\section{ACKNOWLEDGEMENT}

This work was supported by the SPIE ICS-INSA Lyon IoT chair.

\section{REFERENCES}

[1] K. Finkenzeller, RFID handbook: fundamentals and applications in contact-less smart cards and identification. New York, NY, USA: John Wiley and Sons, Inc., 2003, p. 427, (in English).

[2] P. V. Nikitin, R. Martinez, S. Ramamurthy, H. Leland, G. Spiess, and K. V. S. Rao, "Phase based spatial identification of uhf rfid tags," in 2010 IEEE International Conference on RFID (IEEE RFID 2010), April 2010, pp. 102-109.

[3] S. Tedjini, G. Andia-Vera, M. Zurita, R. C. S. Freire, and Y. Duroc, "Augmented rfid tags," in 2016 IEEE Topical Conference on Wireless Sensors and Sensor Networks (WiSNet), Jan 2016, pp. 67-70.

[4] V. Liu, A. Parks, V. Talla, S. Gollakota, D. Wetherall, and J. R. Smith, "Ambient backscatter: Wireless communication out of thin air," SIGCOMM Comput. Commun. Rev., vol. 43, no. 4, pp. 39-50, Aug. 2013. [Online]. Available: http://doi.acm.org/10.1145/2534169.2486015

[5] P. V. Nikitin, S. Ramamurthy, R. Martinez, and K. V. S. Rao, "Passive tag-to-tag communication," in 2012 IEEE International Conference on RFID (RFID), April 2012, pp. 177-184.

[6] J. F. Ensworth and M. S. Reynolds, "Every smart phone is a backscatter reader: Modulated backscatter compatibility with bluetooth 4.0 low energy (ble) devices," in 2015 IEEE International Conference on RFID (RFID), April 2015, pp. 78-85.

[7] Y. Karimi, A. Athalye, S. R. Das, P. M. Djuri, and M. Stanaevi, "Design of a backscatter-based tag-to-tag system," in 2017 IEEE International Conference on RFID (RFID), May 2017, pp. 6-12.

[8] J. Ryoo, J. Jian, A. Athalye, S. R. Das, and M. Stanaevi, "Design and evaluation of bttn: A backscattering tag-to-tag network," IEEE Internet of Things Journal, vol. 5, no. 4, pp. 2844-2855, Aug 2018.

[9] P. V. Nikitin and K. V. S. Rao, "Performance limitations of passive uhf rfid systems," in 2006 IEEE Antennas and Propagation Society International Symposium, July 2006, pp. 1011-1014.

[10] L. Zhou, F. Hutu, G. Villemaud, and Y. Duroc, "Simulation framework for performance evaluation of passive rfid tag-to-tag communications," in 2017 11th European Conference on Antennas and Propagation (EUCAP), March 2017, pp. 500-504.

[11] J. Kabara, P. Krishnamurthy, R. A. Thompson, and D. Tipper, The Physical Layer of Communications Systems. Boston, USA: Artech House., 2006, p. 888, (in English).

[12] P. Z. Peebles, Digital Communication Systems. Harlow, United Kingdom: Prentice Hall., 1986, p. 432, (in English). 